$15^{\text {th }}$ International Conference on

AEROSPACE SCIENCES \& AVIATION TECHNOLOGY,

$\boldsymbol{A S A T}$ - 15 - May 28 - 30, 2013, Email: asat@mtc.edu.eg,

Military Technical College, Kobry Elkobbah, Cairo, Egypt,

Tel: +(202) 24025292 -24036138, Fax: +(202) 22621908

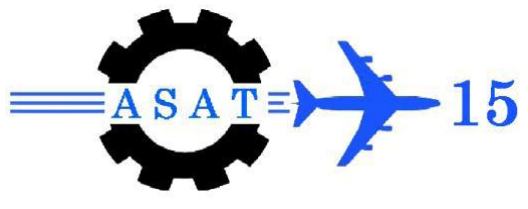

\title{
Simulation of Ship Maneuvering behavior Based on the Modular Mathematical Model
}

\author{
S. Abdel-latif*, M. Abdel-geliel ${ }^{\dagger}$, and E. Eldin Zakzouk ${ }^{\ddagger}$
}

\begin{abstract}
With the rapid development of the computer technology and its successful application in ship engineering, the method of computer simulation based on the mathematical models became more and more popular; it provides a convenient tool for predicting ship maneuverability. One of the preconditions for applying that is the modeling of the dynamic differential equations that represent the ship dynamics in three degrees of freedom. The effectiveness of simulation is guaranteed by how accurate the model is. There are different types of ship mathematical model. In this work, the ship modular mathematical model was investigated. Simulink software was utilized to develop the ship subsystems as individual modules. Modules hydrodynamic forces, and moments were implemented in simulating the ship maneuvering behaviors of the ESSO OSAKA tanker class ship. Moreover, different types of maneuvering are test in particular, turning and zigzag.
\end{abstract}

Keywords: Ship mathematical model, ship dynamic motion, ship maneuvering simulation

\section{Introduction}

Studying the maneuverability of a ship has a great importance in order to avoid collusion with unpredictable objects. Moreover, it helps in determining the ship constraints either in its dynamics or control signal commands.

Various types of mathematical model for maneuvering have been developed by different institutes, they are based on different assumptions either theoretical approaches or experimental techniques [6,7]. However, generally, the mathematical model could be classified into two types; the whole ship model and the mathematical modeling group (MMG) model.

The whole ship model also called "Abkowitz" model [8] is named so after Prof. Abkowitz, 1964 who proposed a method for expressing the hydrodynamic forces and moments by implementing a regression model that treats the maneuvering ship as a complete entity and the forces acting upon it are represented as a Taylor Series in kinematics and geometrical variables (Velocities, accelerations, rudder angle, propeller rate, etc.)

\footnotetext{
Egyptian Armed forces, Egypt; sam_szaz@ hotmail.com

$\dagger$ Associate Professor, Electrical and Control Engineering Department, College of Engineering and Technology, Arab Academy for Science, Technology, and Maritime Transport, Abu-Quir, Alexandria, Egypt; $\underline{m \_a b d e l g l i e l @ y a h o o . c o m ~}$

$\$ \quad$ Professor, Electrical and Control Engineering Department, College of Engineering and Technology, Arab Academy for Science, Technology, and Maritime Transport, Abu-Quir, Alexandria, Egypt; zakzok@aast.edu
} 
The other method (MMG) is proposed by the Japanese Mathematical Modeling Group, (JMMG) in the late 1970s [11,12], In such modular mathematical model; each of the ship individual elements (such as the hull, rudder, propeller, engines and external influences) are considered as separate modules and contribute to the total hydrodynamic forces acting on the ship.

The MMG modeling method is addressed here and applied in Simulink software to model, simulate, and analyze the ship dynamic system. In the proposed Simulink program, each ship subsystem was built as an individual model, and all hydrodynamics nonlinear properties of the ESSO OSAKA tanker class ship [14] were added and modeled in continuous time simulation to predict the ship maneuvering behavior. Two types of maneuvering tests; turning test and zigzag test were performed on the simulated model and the ship behavior results were analyzed.

The paper is organized as follow: section 1 introduces the ship dynamic and kinematic and the ship maneuvering prediction; the mathematical model based on MMG is deduced in section 2; the implementation of the MMG model and the maneuver simulation is explained in section 3; the result analysis is discussed in section 4; and finally the conclusion of the work is summarized.

\section{Ship Dynamics and Kinematics}

In essence a set of equations of motion are constructed based on rigid body dynamics to describe the ship motion in three degree of freedom. These three motions are ; the longitudinal translational motion 'surge motion' produced by the longitudinal force ' $X$ ' , the lateral translational motion 'sway motion' produced by the lateral force ' $Y$ ' , and the rotational motion around the $z$-axis 'yaw motion' produced by the moment around the $z$-axis ' $N$ ', these equations are derived according to the Newton second law, the Newtonian law of motion. Linearization of the general equations is achieved by treating the maneuvering motion of the ship as small deviations from a basic reference motion [1].

\subsection{Coordinate Systems}

To investigate the ship maneuverability by means of mathematical tool, two right-handed coordinate systems are adopted: the earth-fixed (Inertial) coordinate system " $O_{0}-x_{0} y_{0} z_{0}$ " and the body-fixed coordinate system " $O-x y z$ " which moves together with the ship as shown in Fig. 1, the " $O_{0}-x_{0} y_{0}$ " plane and the " $O-x y$ " plane lie on the undisturbed free surface, with the $x_{0}$ axis pointing to the direction of the original course of the ship, whereas the $z_{0}$ axis and the $z$ axis point downwards vertically [2].

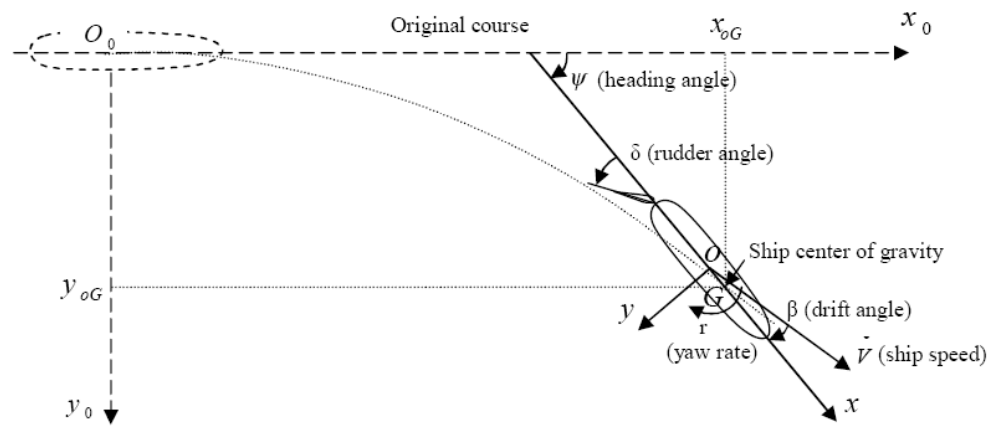

Fig. 1 Coordinate systems 
The angle between the directions of $x_{0}$ axis and $x$ axis is defined as the heading angle " $\psi$ ", at the moment as maneuvering motion is getting start, the two coordinate systems coincide with each other, at any later moment, the position of the ship is determined by the coordinates $x_{O G}$ and $y_{0 G}$ of the ship center of gravity in the earth-fixed coordinate system, and the orientation of the ship is determined by the heading angle " $\psi$ ". The maneuvering motion of the ship in the horizontal plane is described by " $V$ " the speed of translational motion and " $r$ " the yaw rate of rotational motion about the $z$ axis. The components of the speed " $V$ " in the directions of $x$ axis and $y$ axis are " $u$ " and " $v$ " respectively. The angle between the directions of speed " $V$ " and $x$ axis is defined as the drift angle " $\beta$ ", obviously we have $u=V \cos \beta$, and $\quad v=-V \sin \beta$

The earth-fixed (Inertial) coordinate system is an inertial frame in which the Newtonian law of motion is valid. In this coordinate system, we have the equations of motion as follows;

where;

$$
\begin{aligned}
X_{O} & =m \ddot{x}_{O G} \\
Y_{O} & =m \ddot{y}_{O G} \\
N_{O} & =I_{z} \ddot{\psi}_{O G}
\end{aligned}
$$

$X_{o}, Y_{o}, N_{o}$ are the components of external force acting on the ship in the directions of $\mathrm{x}_{0}$ axis, $\mathrm{y}_{0}$ axis , and the moment about the $\mathrm{z}$ axis respectively.

$m \quad$ is the mass of the ship.

$I_{z} \quad$ is the moment of inertia of the ship about $\mathrm{z}$ axis.

$\psi \quad$ is the yaw acceleration.

$\ddot{x}_{O G}, \ddot{y}_{O G}$ are the components of acceleration in $\mathrm{x}_{0}$ axis and $\mathrm{y}_{0}$ axis directions, respectively.

\subsection{Axis Transform}

For purpose of ship maneuver prediction, it is more convenient to use the equations of motion in the body fixed system $[x, y, z, u, v, X, Y, N]$ instead of the earth-fixed inertial system $\left[x_{0}\right.$ $\left., y_{0}, z_{0}, u_{0}, v_{0}, X_{0}, Y_{0}, N_{0}\right]$. The relations between the kinematics parameters defined in the earth-fixed and the body-fixed coordinate systems are to be utilized [1,2]. With the original of the body-fixed coordinate system lying on the center of gravity.

$$
\begin{aligned}
& x_{0}=x_{O G}+x \cos \psi-x \sin \psi \\
& y_{0}=y_{O G}+x \sin \psi+y \cos \psi \\
& z_{0}=z
\end{aligned}
$$

assume that $\quad x_{0}{ }^{\prime}=x_{0}-x_{0 G}, y_{0}{ }^{\prime}=y_{0}-y_{0 G}$, and $z_{0}{ }^{\prime}=z_{0} \quad$ Then;

$$
\begin{aligned}
& x_{0}^{\prime}=x \cos \psi-y \sin \psi \\
& y^{\prime}{ }_{0}=x \sin \psi+y \cos \psi \\
& z^{\prime}{ }_{0}=z
\end{aligned}
$$

we can rewrite the components of the body system and the components of the inertial system as the elements of the vectors $[X]$ and $\left[X_{0}\right]$ respectively, the relationship between the two system as shown in Fig. 2.

$$
[\mathrm{X}]=[\mathrm{R}]\left[\mathrm{X}_{0}\right] \text {, and }\left[\mathrm{X}_{0}\right]=[\mathrm{R}]^{\mathrm{T}}[\mathrm{X}]
$$

where ;

$[R]$ is the transform matrix

$$
[R]=\left(\begin{array}{cc}
\cos \psi & \sin \psi \\
-\sin \psi & \cos \psi
\end{array}\right)
$$

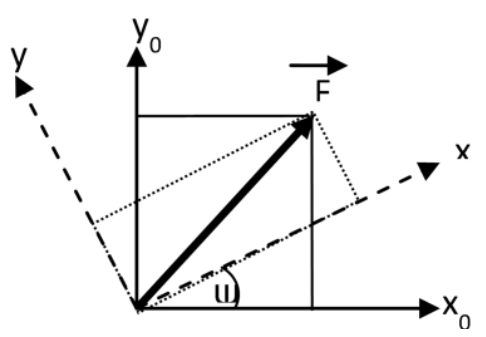

Fig. 2 Axis transform 
The forces in the directions of $x$ axis and $y$ axis by $X$ and $Y$, respectively, is expressed by:

$$
\begin{aligned}
& X=X_{0} \cos \psi+Y_{0} \sin \psi \\
& Y=-X_{0} \sin \psi+Y_{0} \cos \psi
\end{aligned}
$$

The components of ship speed in the directions of $x$ axis and $y$ axis by $u_{G}$ and $v_{G}$ respectively, are;

$$
\begin{aligned}
& \dot{x}_{O G}=u_{G} \cos \psi-v_{G} \sin \psi \\
& \dot{y}_{O G}=u_{G} \sin \psi+v_{G} \cos \psi
\end{aligned}
$$

Differentiating Equation (5) with respect to time yields

$$
\begin{aligned}
& \ddot{x}_{O G}=\dot{u}_{G} \cos \psi-u_{G} \dot{\psi}_{\sin \psi-\dot{v}_{G} \sin \psi-v_{G} \dot{\psi} \cos \psi} \\
& \ddot{y}_{O G}=\dot{u}_{G} \sin \psi+u_{G} \dot{\psi} \cos \psi+\dot{v}_{G} \cos \psi-v_{G} \dot{\psi} \sin \psi
\end{aligned}
$$

Substituting Equations (1) and (6) into Equation (4), we obtain the equations of motion in the body-fixed coordinate system in the form

$$
\begin{aligned}
& X=m\left(\dot{u}_{G}-v_{G} \dot{\psi}\right) \\
& Y=m\left(\dot{v}_{G}-u_{G} \dot{\psi}\right)
\end{aligned}
$$

Since the moment about the $z$ axis through the center of gravity is same in the body-fixed coordinate system as in the earth-fixed coordinate system $[3,4,5]$, we have the equation of yaw motion in the body-fixed coordinate system as Equation (1), in practice it the origin of the body-fixed coordinate system does not lie on the center of gravity, but on the midship point, while in the body-fixed coordinate system the origin is lying on the midship point, the center of gravity has the coordinates $\left(x_{G}, 0, z_{G}\right)$. In such a coordinate system the components of ship speed at the center of gravity are $u_{G}$ and $v_{G}$, and at the original are $u$ and $v$, where $u_{G}=u$ and $v_{G}=v+x_{G} \dot{\psi}$, therefore, the equations of motion in the body-fixed coordinate system with the original lying on the midship point is given in the form

$$
\begin{aligned}
& X=m\left(\dot{u}-v \dot{\psi}-x_{G} \dot{\psi}^{2}\right) \\
& Y=m\left(\dot{v}+u \dot{\psi}+x_{G} \ddot{\psi}\right) \\
& N=I_{Z} \ddot{\psi}+m x_{G}(\dot{v}+u \dot{\psi})
\end{aligned}
$$

Recalling " $r=\dot{\psi}$ ", we have the equations of motion in the body-fixed coordinate system, with the original coordinate system lying on the midship point, in the form

$$
\begin{aligned}
& X=m\left(\dot{u}-v r-x_{G} r^{2}\right) \\
& Y=m\left(\dot{v}+u r+x_{G} \dot{r}\right) \\
& N=I_{Z} \ddot{\psi}+m x_{G}(\dot{v}+u r)
\end{aligned}
$$

These equations are the Euler equation of motion of rigid body in 3 DOF including the angular momentum terms for the case of origin for motion is not on the centre of gravity

\subsection{Maneuver Prediction}

Ship motion differential equations, can be solved by numerical methods such as the RungeKutta method to determine the components of maneuvering motion $u(t), v(t)$ and $r(t)$ at any time[8] ,the simulating program represents the Euler's equations of motion, and the MMG ship mathematical model previously described early in chapter two, the inputs to the program 
are the hydrodynamic coefficients of the ship in three degrees of freedom and the ship parameters used to evaluate the hydrodynamic forces and moment acting on the ship hull (surge, sway, and yaw), and by using the proposed maneuvering prediction method the accelerations and velocities are obtained and fed into the axis transform module and output the ship's current position $\left(x_{s}, y_{s}\right)$ and the heading angle $\left(\psi_{s}\right)$.

The position and the orientation of the ship can be determined by the coordinates of the center of gravity in the earth-fixed coordinate system and the heading angle of the ship, which can be obtained by numerical integrations as follows ;

$$
\begin{aligned}
x_{0 G}(t) & =\int_{0}^{t} \dot{x}_{0 G}(t) d t ; \\
y_{0 G}(t) & =\int_{0}^{t} \dot{y}_{0 G}(t) d t ; \\
\psi_{0 G}(t) & =\int_{0}^{t} \dot{\psi}_{0 G}(t) d t ;
\end{aligned}
$$

where:

$$
\begin{gathered}
\dot{x}_{0 G}(t)=u(t) \cos \psi-\left[v(t)+x_{G} r(t)\right] \sin \psi(t) \\
\dot{y}_{0 G}(t)=u(t) \sin \psi(t)+\left[v(t)+x_{G} r(t)\right] \cos \psi(t) \\
\dot{\psi}(t)=r(t)
\end{gathered}
$$

Prediction of the ship maneuvering can be achieved by obtaining the ship position in the earth coordinate, and the heading direction at each time instant, and any standard maneuvers can be simulated by a computer and the maneuvering characteristics of the ship can be predicted, , the equations used in the simulation are the Euler equation previously described in equation (9), the surge force expressed by:

$$
X=m\left(\dot{u}-v r-x_{G} r^{2}\right)
$$

The equation was rearranged in order to the set of acceleration form that acceleration due to surge force that need to be integrated to obtain velocities and displacements are:

$$
\dot{u}=(X / m)+v r+x_{G} r^{2}
$$

Solving equation (12) by numerical integration in the time domain using the ODE function and the velocity of the ship in time domain can be obtained by single integration as follows:

$$
u(t)=\int \dot{u}(t) d t=\int\left[(X / m)+v r+x_{G} r^{2}\right] d t
$$

And the displacement of the motion could be obtained with double Integration.

$$
x_{O G}=\int u(t) d t=\iint_{[}\left[(X / m)+v r+x_{G} r^{2}\right] d t
$$

Similarly, by implementing the numerical integration in time domain on the Euler equations for the sway force and yaw moment, the ship displacement in the $\mathrm{y}$ axis $\left(\mathrm{y}_{\mathrm{OG}}\right)$ and the turning angle $\left(\psi_{s}\right)$ for the ship heading.

$$
\begin{aligned}
\mathrm{y}_{\mathrm{OG}}=\int \mathrm{v}(\mathrm{t}) \mathrm{dt}=\iint\left[\left(\mathrm{Y} / \mathrm{m}-\mathrm{ur}-\mathrm{x}_{\mathrm{G}} \mathrm{r}\right)\right] \mathrm{dt} \\
\quad \psi_{s}=\int r(t) d t=\iint\left[\left(N / I z-m x_{G} / I z(v+u r)\right] d t\right.
\end{aligned}
$$




\section{Ship Mathematical Model}

To simplify the scope of the work, the MMG mathematical model has been adopted ; the MMG type model separates the forces experienced by the hull, propeller, and rudder and also includes the interaction effects between the components of model that could be developed and tested separately $[11,12]$, the model individual elements hydrodynamic forces and moments acting on the ship can be divided into the following components;

$$
\begin{aligned}
& X=X_{H}+X_{P}+X_{R} \\
& Y=Y_{H}+Y_{P}+Y_{R} \\
& N=N_{H}+N_{P}+N_{R}
\end{aligned}
$$

where;

The subscripts $\mathrm{H}, \mathrm{P}$, and $\mathrm{R}$ denote the hull, the propeller, and the rudder respectively,

The advantage of using the modular type model is that changing the parameters of an individual module doesn't altering other modules, e.g. a change in rudder size or propeller geometry could be done without having to change other modules.

For simulation purpose the proposed ship model in this study used the specifications, characteristics, as well as the hydrodynamic coefficients of commercial Esso tanker class

\begin{tabular}{|c|c|c|c|c|c|c|c|c|c|}
\hline $\mathrm{m}$ & 3500000 & $\mathrm{~N} \mathrm{~V}$ & 98631.9 & $\begin{array}{l}\mathrm{X} \\
\mathrm{VV}\end{array}$ & 20567 & $\begin{array}{ll}\mathrm{X} & \mathrm{VVVVV}\end{array}$ & 94225 & $\mathrm{Xg}$ & 50 \\
\hline$m x$ & 523500 & $\mathrm{~N} r$ & -1293619 & $\mathrm{X}$ vr & 79987 & Y VVr & 47866 & $\mathrm{~N}$ VVr & 86003 \\
\hline my & 533700 & $\mathrm{Y}=\mathrm{V}$ & -159684 & Y_rrr & -41239 & $\mathrm{~N}$ rrr & 2667498 & $\mathrm{~N}$ vrr & 27866 \\
\hline Iz & 56470 & Y_r & -119712 & $Y \_V V V$ & 79871 & $\mathrm{~N} \_\mathrm{VVV}$ & -6423869 & & \\
\hline
\end{tabular}
ship[14], Table 1 illustrates the parameters, Hydrodynamic coefficients of used in this study.

Table 1 Parameters and Hydrodynamic Coefficients

\subsection{Hydrodynamic Forces Acting on the Hull}

For the hull module, the hull forces could be represented by the hydrodynamic derivatives model that represents the hull characteristic such as hull-form geometry, the hydrodynamic forces and moment acting on the hull are expressed by the components $X_{H}, Y_{H}$, and $N_{H}$ as in the following equations :

$$
\begin{aligned}
& X_{H}=-m_{x} u+R_{T}(u)+X_{v v} v^{2}+\left(X_{v r}-m_{y}\right) v r+X_{r r} r^{2}+X_{v v v v} v^{4} \\
& Y_{H}=-m_{y} v+Y_{v} v+\left(Y_{r}-m_{x}\right) r+Y_{v v v} v^{3}+Y_{v v r} v^{2} r+Y_{v r r} v r^{2}+Y_{r r r} r^{3} \\
& N_{H}=-I_{z} r+N_{v} v+N_{r} r+\left(11 y v v v^{3}+N_{v v r} v^{2} r+N_{v r r} v r^{2}+N_{r r r} r^{3}\right.
\end{aligned}
$$

where;

$m_{x}, m_{y} \quad$ are the added masses in the $\mathrm{x}$, and $\mathrm{y}$ direction

$R_{T}(u) \quad$ is the resistance force $R_{T}(u)=a_{0}+a_{1} u+a_{2} u^{2}$

$I_{z} \quad$ is the moment of inertia about the $\mathrm{z}$ axis

$X_{v v}, X_{v v v v} \quad$ are Non-linear components of surge force due to sway motion

$X_{v r}$

$X_{r r}$ is the Coupling coefficient of sway motion and yaw motion for surge force

$Y_{v}, Y_{v v v}$

$Y_{v v r}, Y_{v r r}$

$Y_{r r r}$

$N_{v}$

$N_{v v v}$ is the Non-linear component of surge force due to yaw motion is the Non-linear component of sway force due to sway motion are the NL Coupling coefficient of sway and yaw motion for sway force is the Non-linear component of sway force due to yaw motion is the yaw moment due to sway motion

$N_{v v r}, N_{v r r}$ $N_{r r r}$ is the Non-linear component of yaw moment due to sway motion. are the NL Coupling coefficient of sway and yaw for yaw moment. is the NL component of yaw moment due to yaw 


\subsection{Hydrodynamic Forces Induced by Propeller}

Calculation of propulsive forces is modeled with respect to the thrust system used in the ship whether it is propeller or water jet. The calculation of propulsive forces therefore is dependent upon accurate representation of wake, lifting and drag or more generally in the context of propeller-hull-rudder interaction as it is mentioned in the previous section. In the current model, propulsive forces are calculated using formulations in Inoue et al. (1981) and Spyrou (1990) for the propeller system [13].

$$
\begin{aligned}
& X_{P}=(1-t) \rho K_{T} D_{P}^{4} n^{2} \\
& Y_{P}=\rho n^{2} D^{4} Y_{P}{ }^{*} \\
& N_{P}=\rho n^{2} D^{5} N_{P}
\end{aligned}
$$

where;

$K_{T} \quad$ is the thrust coefficient of the propeller and is described by the function of advance ratio $(J)$ as : $K_{T}=a_{1}+a_{2} J+a_{3} J^{3}$ where; $J=u_{P} / n D_{P}$, and $u_{P}=u\left(1-W_{P}\right)$

$W_{P} \quad$ wake fraction coefficient

(1-t) thrust deduction factor (interaction coefficient between hull \& propeller)

$D_{P} \quad$ propeller diameter

$u_{P} \quad$ inflow velocity of the propeller

$n \quad$ propeller revolution rate

$a_{1}, a_{2}, a_{3}$ are constants for propeller open characteristics

$Y_{P}{ }^{*}, N_{P} *$ are generally dependent on $u / n \cdot p$; as $p$ is the propeller pitch

$Y_{P}, N_{P}$ have small effect on the ship motion thus they can be neglected

\subsection{Hydrodynamic Forces Induced by the Rudder}

As an important element of the excitation for ship motions in following and quartering seas, the forces on a rudder, when considered as a separate individual element, can be obtained with the derivation of the lift and drag generated from the rudder.

Performance of rudder is greatly influenced by the interactions between rudder to hull and rudder to propeller, due to the change of the lift [10].

Based on the above background, Inoue et al. (1981), adopted a formula for calculating the rudder forces including the aforementioned interactions that later was used in the MMG model, the rudder forces and moments including rudder to-hull interaction are as follows:

$$
\begin{aligned}
& X_{R}=-\left(1-t_{R}\right) F_{N} \sin \delta \\
& Y_{R}=-\left(1+a_{H}\right) F_{N} \cos \delta \\
& N_{R}=-\left(x_{R}+a_{H} x_{H}\right) F_{N} \cos \delta
\end{aligned}
$$

where;

$t_{R} \quad$ is the interactive force coefficient between Hull and rudder

$a_{H} \quad$ is the rudder to Hull interaction coefficient

$x_{H} \quad$ is the longitudinal coordinate of interaction force point of action

$x_{R} \quad$ is the longitudinal coordinate of rudder center of pressure $=(-L / 2)$

$\delta \quad$ is the rudder angle

$F_{N} \quad$ is the rudder normal force and is given by; $F_{N}=\rho / 2 A_{R} f_{\alpha} u_{R}^{2} \sin \left(\alpha_{R}\right)$

$A_{R} \quad$ is the rudder area

$f_{\alpha} \quad$ is the gradient of the lift coefficient of the rudder and is given by the (Fuji's) prediction formula; $f_{\alpha}=6.13 K_{R} /\left(K_{R}+2.25\right)$

$K_{R} \quad$ is the rudder aspect ratio

$u_{R} \quad$ is the longitudinal inflow velocity of the rudder

$\alpha_{R} \quad$ is the effective rudder inflow angle and is given by; $\alpha_{R}=\delta-\beta ;$ as $\beta$ is the drift angle 


\section{Model Implementation}

Simulation of the ship maneuver behavior by changing the ship control parameter is achieved by using MATLAB Simulink module programming; a simulation program was developed to simulate the maneuvering of ship in three degrees of freedom. As a consequence, three Simulink modules were developed to express the ship subsystems representing the ship hull ,propeller, rudder modules, the outputs of all modules are summed and fed back to the ship motion equation module that represents the Euler's equations of motion previously described, and by using maneuvering prediction method previously discussed ,the accelerations and velocities are obtained and fed back ,these feedback values will contribute to the total force value at the next computation time step.

The simulated control parameter representing the rudder and the propeller rotational rate are presented as a variable slide gain element to simulate the navigator maneuvering commands and be monitored by a digital display and a rpm gauge, the output of the ship model as described above are the position of the ship in the earth fixed coordinate ( $\mathrm{x}_{\mathrm{OG}}, \mathrm{y}_{\mathrm{OG}}$ ) which read out by a digital displays and plotted by a X Y plot to indicate the ship motion , and the ship heading angle $\left(\psi_{s}\right)$ which shown as a direction of a analog compass.

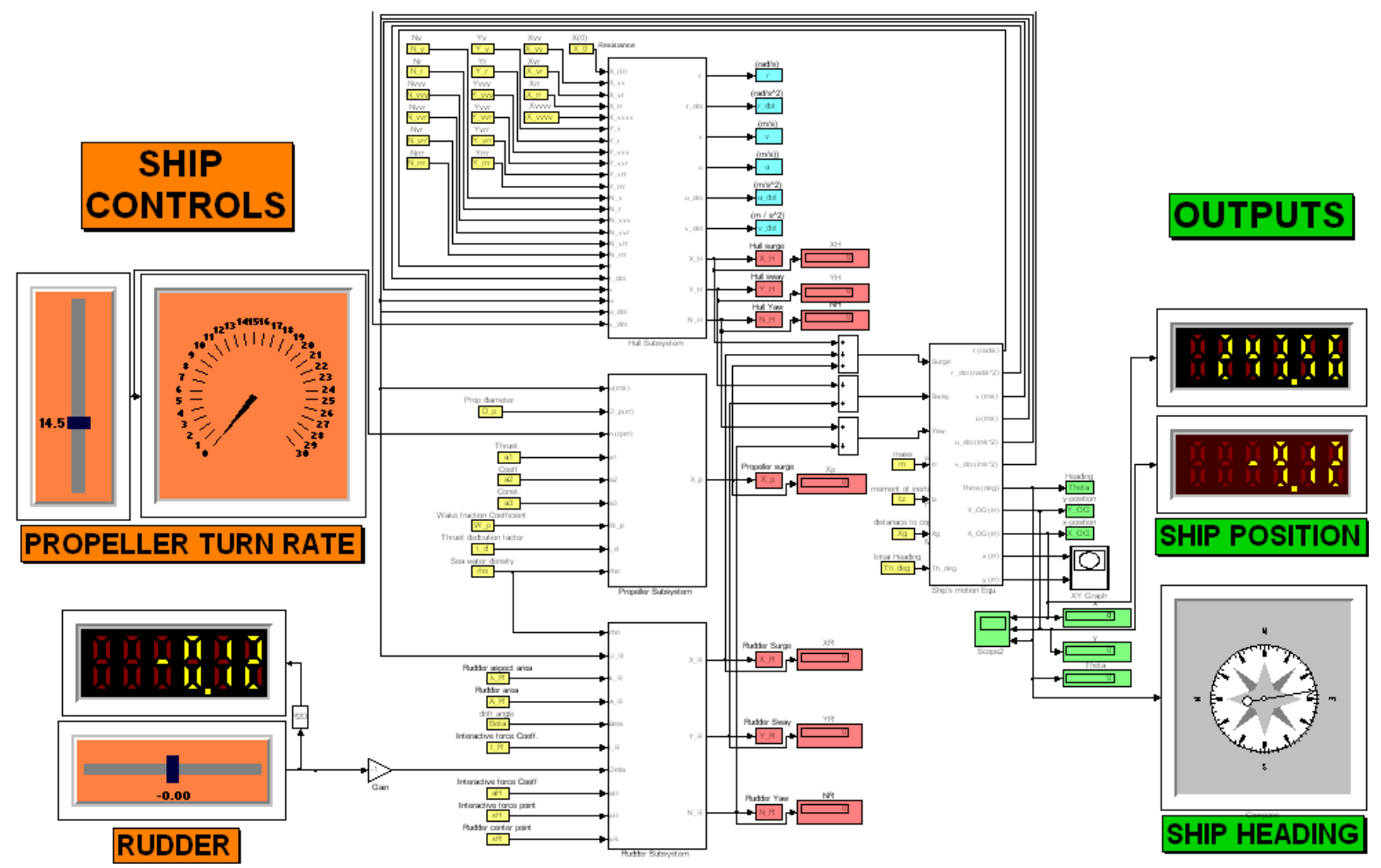

Fig. 4 Simulink Ship MMG model

\subsection{Ship Motion Module}

As shown in Fig. 5, the inputs to the module are the 3DOF hydrodynamic forces and moment (surge, sway, and yaw), the module represents the Euler's equations of motion, and by using maneuvering prediction method, the accelerations and velocities are obtained and fed into the axis transform module and output the ship's current position $\left(x_{s}, y_{s}\right)$ and the heading angle $\left(\psi_{s}\right)$. 


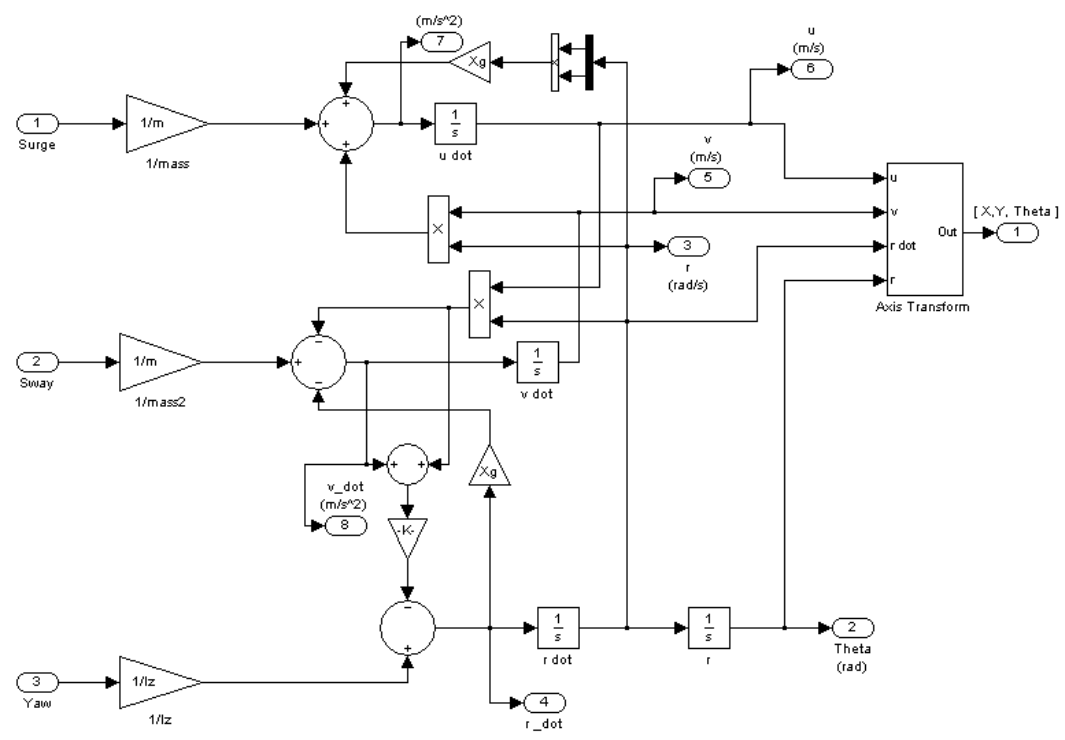

Fig. 5 Ship motion module

The ship characteristics mass $(m)$, moment of inertia $\left(I_{z}\right)$, the distance to the center of gravity $\left(x_{G}\right)$ of the case studied of the ESSO OSAKA tanker class ship are inputted as reference values, the outputs of the modules include the surge, sway, and the yaw angular velocity and accelerations, they all are fed back to the three modules representing the Hull, Propeller, and Rudder subsystem.

\subsection{Hull Subsystem}

The hull subsystem represents the hull characteristic. the hydrodynamic forces and moment acting on the hull are explained in the equation (11) which expresses the forces $X_{H}, Y_{H}$, and $N_{H}$ with regard to the hydrodynamic coefficients induced by the forces and moments acting on the hull body frame ,these coefficients are obtained from the case studied of the ESSO OSAKA tanker class ship [14], the values of the ship coefficients and hull characteristics are fed as input data into the module, accelerations and velocities are inputted to the module as a feedback from the ship motion equation module ,the block diagram of the Hull module is shown in Fig. 6.

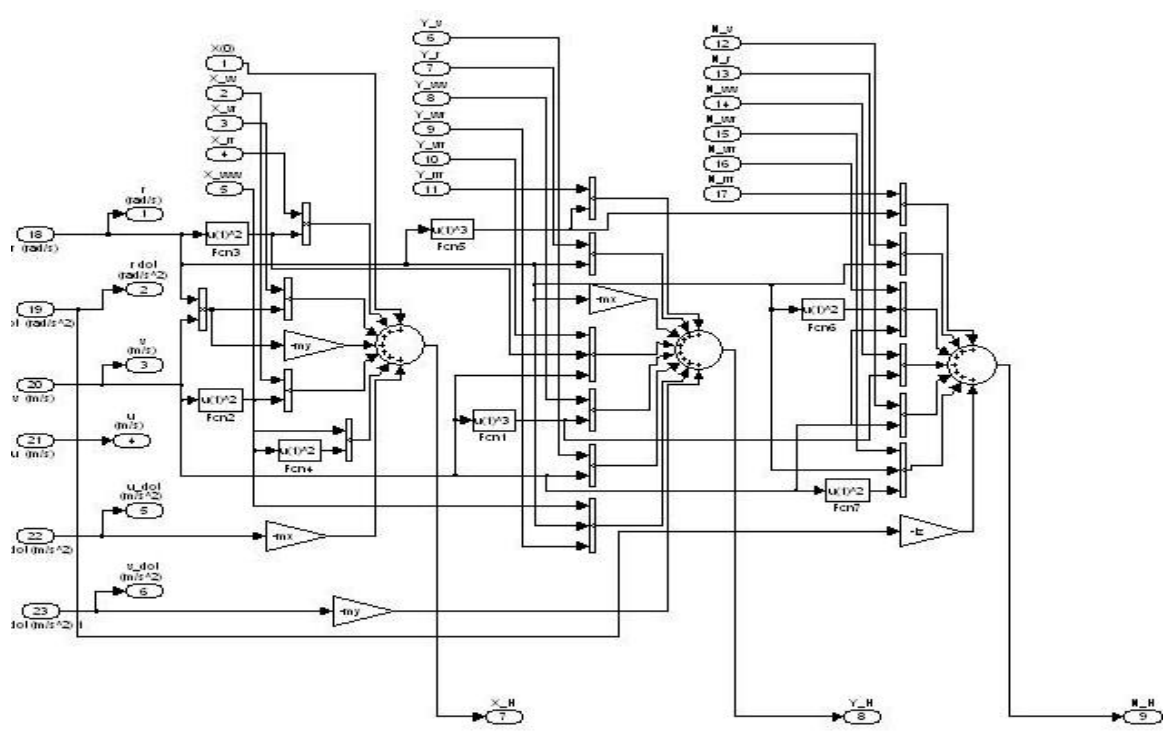

Fig. 6 Hull subsystem module 


\subsection{Propeller Subsystem}

Surge ,sway forces and the yaw moment induced by the ship propeller is denoted by $X_{P}, Y_{P}$, and $N_{P}$, the most influenced force produced by the propeller and cause the ship motion in the translational force is the surge force $X_{P}$ shown in Fig. 7, the propeller subsystem represents the mathematical model of the ship thrust propeller based on the calculation of the propulsive forces using the formulations in Inoue et.al., Equation (12).

The effect of the propeller on the translational lateral motion and the rotation of the ship is very small, thus the sway force $Y_{P}$ and the yaw moments $N_{P}$ induced by the propeller can be neglected. The coefficients of propeller thrust is obtained from predetermined experimental data, other propeller characteristics are empirically estimated and inputted to the propeller subsystem module.

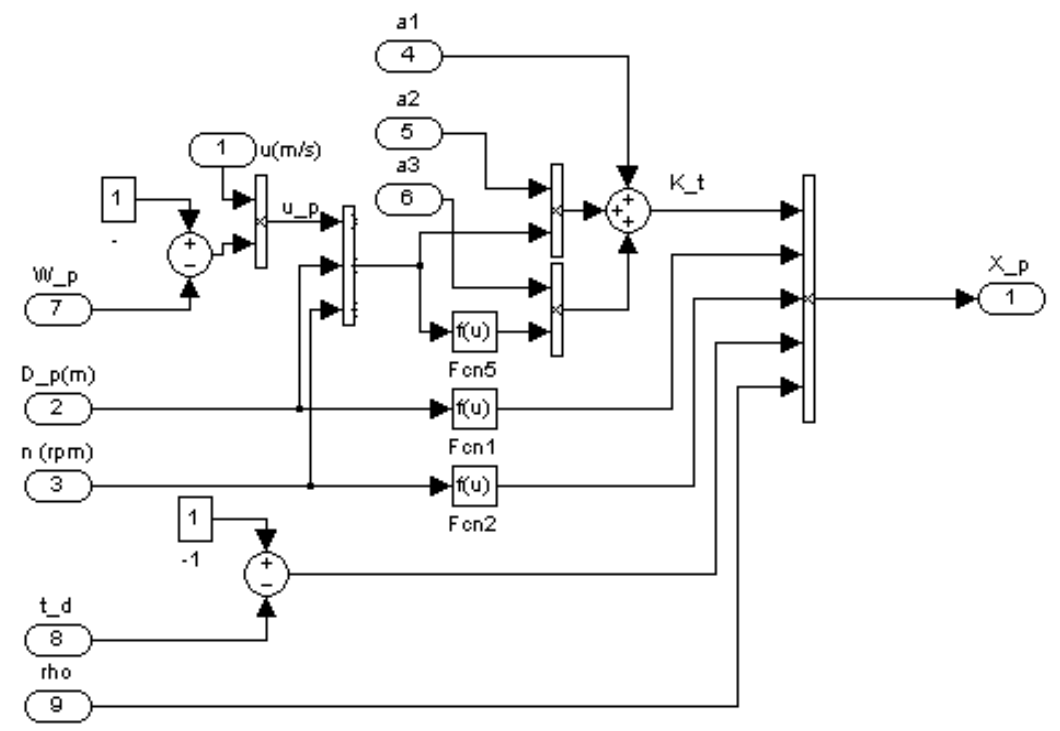

Fig. 7 Propeller Subsystem module

\subsection{Rudder Subsystem}

The forces on a rudder, when considered as a separate individual element, can be obtained with the derivation of the lift and drag generated from the rudder. Performance of rudder is greatly influenced by the interactions between rudder to hull and rudder to propeller, due to the change of the lift, the rudder subsystem module represents the mathematical model of the rudder and interactive forces with the hull.

Forces and moment induced by the Rudder $X_{R}, Y_{R}$, and $N_{R}$ are expressed as the formula in equation (14) as shown in Fig. 8, the interaction coefficients between the hull and the rudder, the rudder geometric dimensions, and constant parameters along with the drift angle and the rudder control command expressed as the rudder angle $(\delta)$; all the above mentioned data is obtained from predetermined experimental tests or evaluated as estimated parameters inputted to the module. 


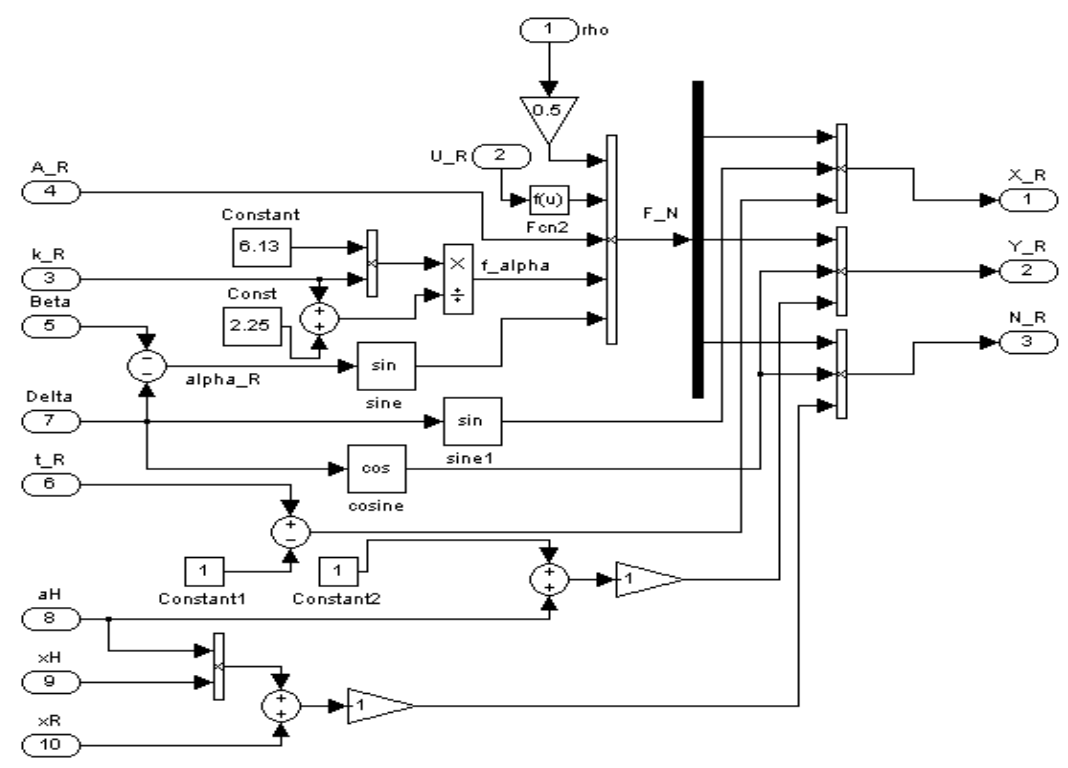

Fig. 8 Rudder subsystem module

\section{Model Simulation Results}

The accuracy of the simulation of ship behavior depends mainly on the predefined hydrodynamic coefficients and the precision of modeled ship parameters, and technical data.

The ship control parameters are the rudder angle denoted as $(\delta)$ and the propeller turn rate denoted as (n), the rudder angle is bounded by the maximum rudder angle, it was assumed that the pilot action to change the rudder angle could take different shape like actions depends on the required maneuver, therefore the rudder angle control parameter is checked for the following test maneuvers

\subsection{Turning Maneuver Test}

The turning test is performed to evaluate the ship's turning ability, a turning maneuver is to be performed to both starboard and port sides with the maximum design rudder angle permissible at the test speed, the rudder angle is executed following a steady approach with zero yaw rates.

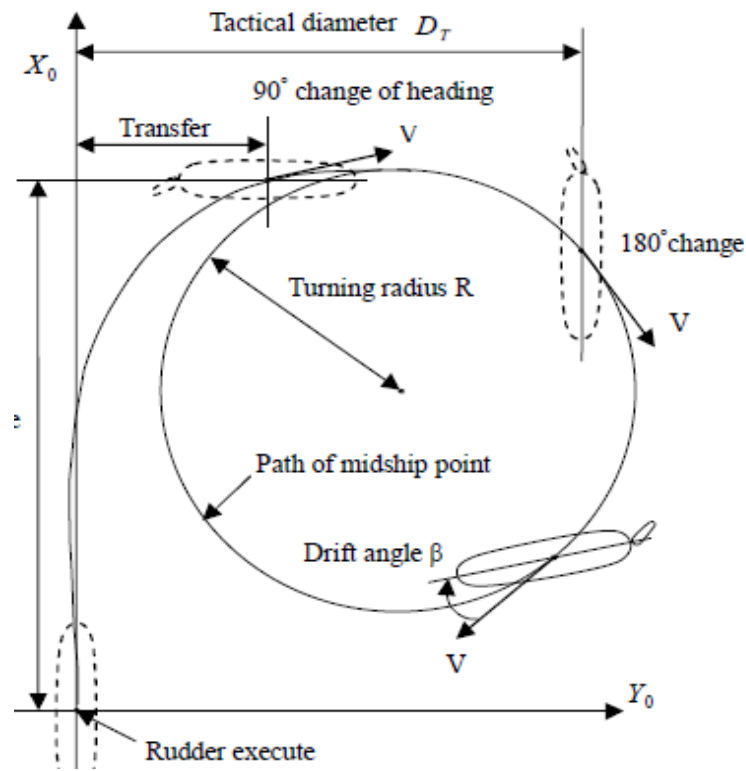

Fig. 9 Turning test maneuver 
The turning test is applied by simulating the rudder angle as a unit step like action that change the rudder angle from 0 to 20 degree and keep the applied angle to the end of simulation time, the step like action results in the ship turning maneuver by changing her heading to the starboard side for a full turn with the applied rudder command [1], the actual turning test maneuver is shown in Fig. 9.

The simulated turning maneuver for a ship running with a propeller turn rate equals to (11rps) revolution per second, and applying a rudder angle in a step like action of 20 degree to the starboard side as shown in Fig. 10, the ship behavior is very much close to the actual turning maneuver

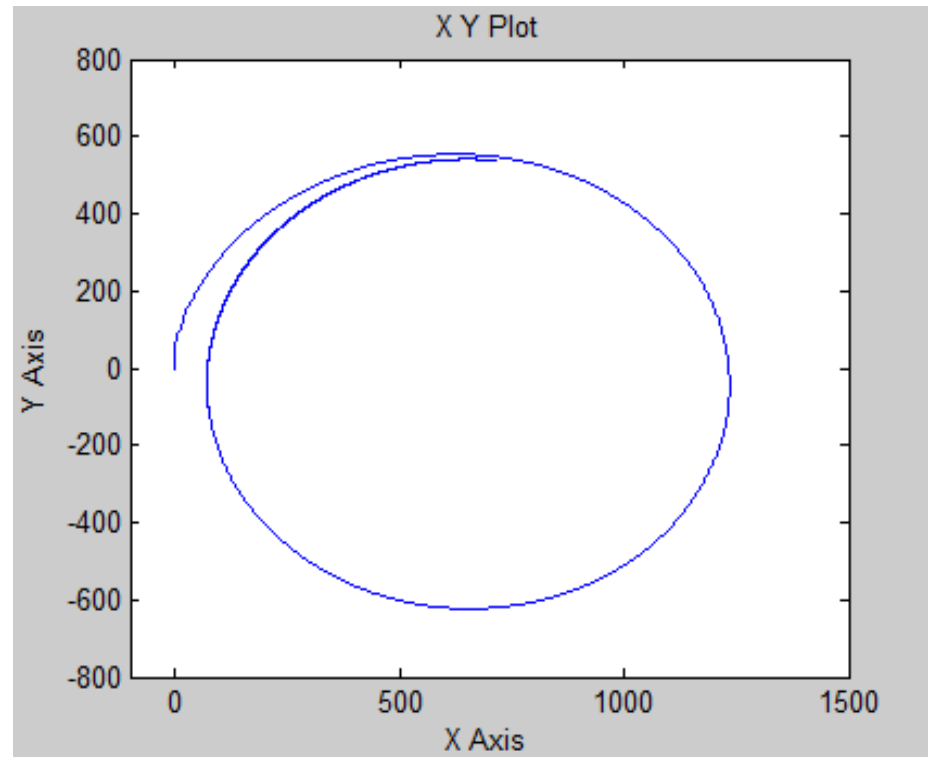

Fig. 10 Model turning maneuver

\subsection{Zigzag Test}

The zigzag test is performed to evaluate the initial turning, the yaw-checking and the coursekeeping abilities. A zigzag test is to be initiated to both starboard and port and begins by applying a specified amount of rudder angle. The rudder angle is then alternately shifted to either side after a specified deviation from the original heading of the ship is reached [1].

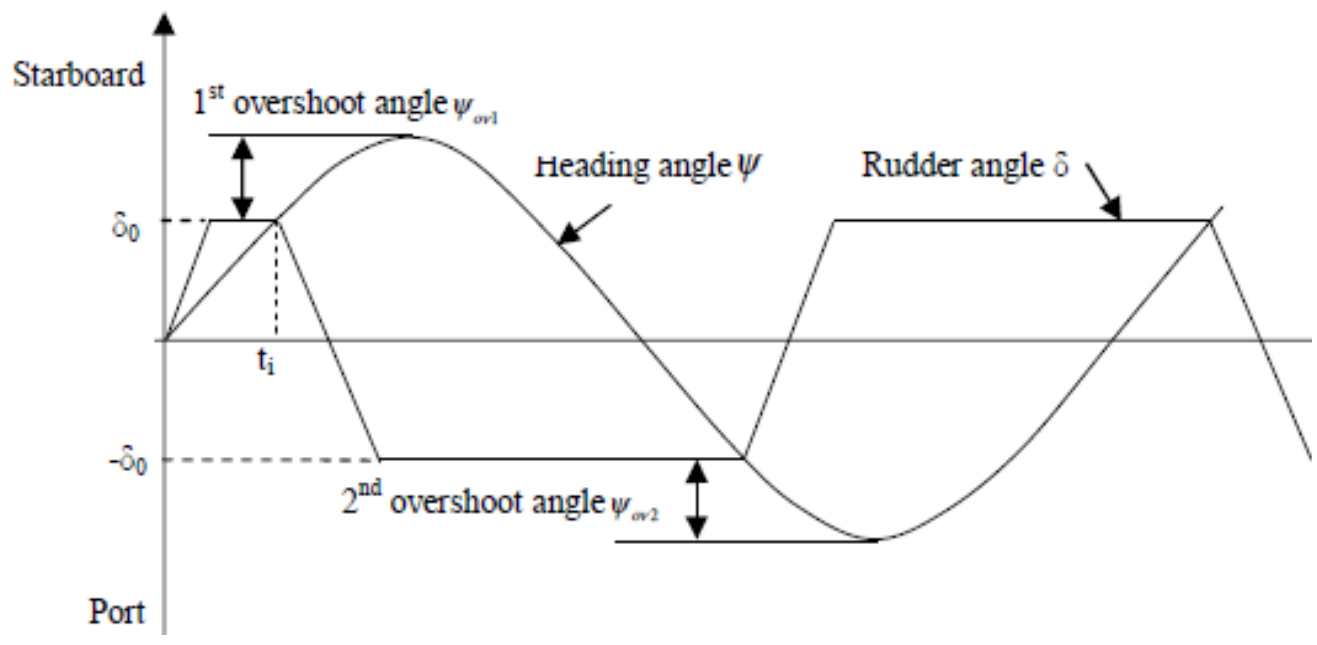

Fig. 11 Zigzag test maneuver 
There are two kinds of zigzag tests, the $10^{\circ} / 10^{\circ}$ and $20^{\circ} / 20^{\circ}$ tests, the $10^{\circ} / 10^{\circ}$ test uses $10^{\circ}$ rudder angles to either side following a heading deviation of $10^{\circ}$ from the original course. The $20^{\circ} / 20^{\circ}$ zigzag test uses $20^{\circ}$ rudder angles and $20^{\circ}$ heading deviation from the original course as shown in Fig. 11, the model test is simulated by applying a generated signal similar to the pulse shown in Fig. 12 that reflects the rudder action of $20^{\circ} / 20^{\circ}$ zigzag maneuver

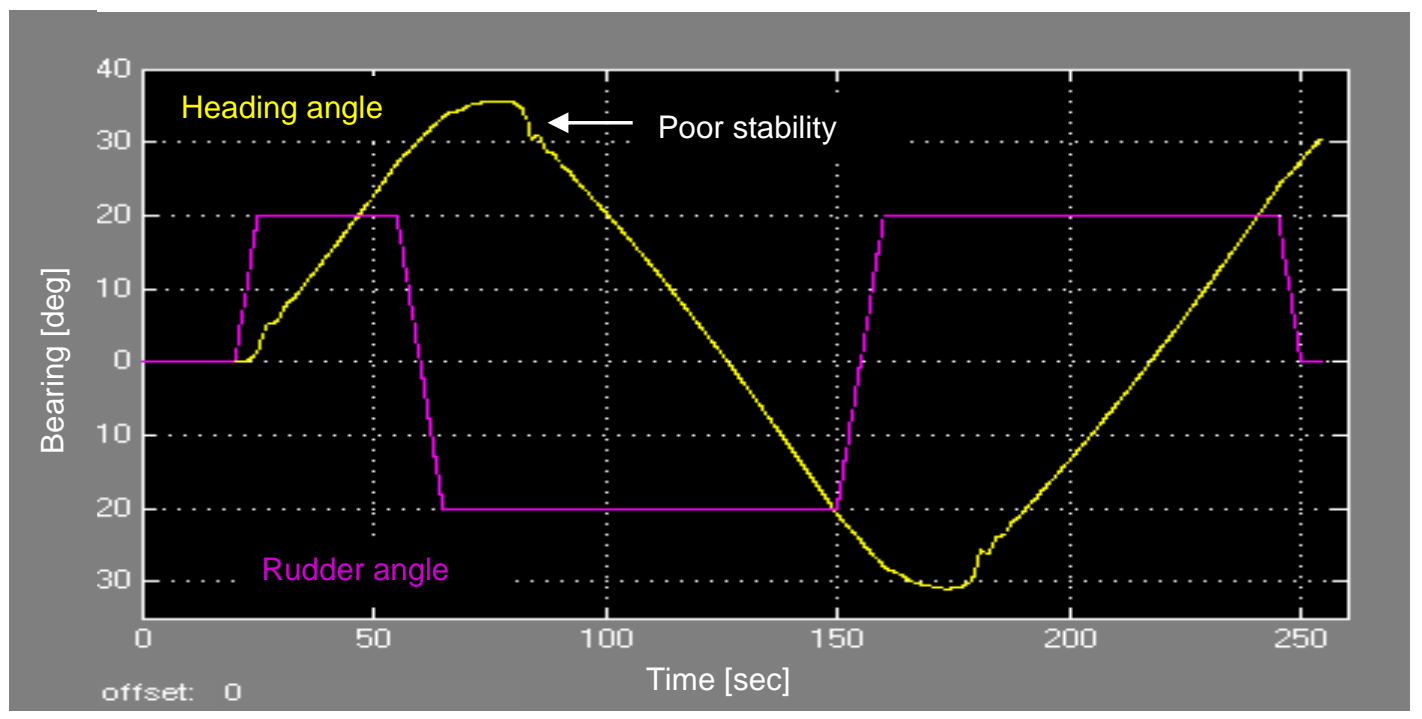

Fig. 12 Model zigzag maneuver

The accumulated errors of the empirical formulas used in the calculations along with the inaccuracy of the parameters, and hydrodynamic coefficients of ship model causes a dynamically unstable ship model or a model with poor dynamic stability this instability can be noticed while performing the zigzag maneuvering test.

\section{Conclusion}

One of specific characteristics of MMG model is to express the hydrodynamic forces caused by propeller and rudder and interaction among hull, propeller and rudder correctly based on the hydrodynamic phenomenon. There are several constants in the proposed model mentioned above that have an important effect on the ship maneuvering motion. The use of the different values of hydrodynamic coefficients has an extremely important effect on the ship maneuverability and stability.

The ship modular mathematical model proposed in the study used to simulate the ship behavior versus different maneuvers that can be used to investigate the different effects caused by application of the surge longitudinal force $\mathrm{X}$, the sway lateral force $\mathrm{Y}$, and yaw rotating moment $\mathrm{N}$ on the turning and zigzag motion estimation, measuring the ship turning ability by obtaining the ship tactical parameters such as the tactical diameter, the advance, and the transfer distance can also be determined, time histories of the rudder angle, turning angle, overshoot angles due to zigzag maneuvering, the influence of the change of ship's speed and the difference in drifting angle can also be investigated.

The ship mathematical model represented in the study is under condition of calm and deep water conditions, in the actual navigational situation, there are many kinds of environmental forces such as wind, wave and current have an extended effect on the ship. These 
environmental forces have an influence on the dynamic behavior of the ship maneuver. It will be proper in the future to investigate the mathematical formulas of such forces and add them as additional modules

\section{References}

[1] Dr.-Ing.Zou Zaojian "Ship Maneuvering and Sea keeping",School of naval Architecture, Ocean and civil engineering, Shanghi Jiao Tong University, April 2006.

[2] ChingYawTzeng ,Ju-Fen Chen "Fundamental properties of linear ship steering dynamic model”, National Taiwan Ocean University, Keelung ,Taiwan ,R.O.C , Journal of Marine Science and Technology,Vol.7,No.2,pp.79-88(1999).

[3] J. GOCLOWESKI, and A. GELB "Dynamics of an Automatic Ship Steering System.", IEEE Transactions on Automatic control, Vol., AG 11,No. 3, July,1996

[4] Zafer Ayaz,Dracos Vassalos ,and Kostas J. Spyrou “ Maneuvering behavior of ships in extreme astern seas" , Naval and Marine Architecture ,Glasgow, Scotland, Ocean Engineering 33-(2006) 2381-2434.

[5] Dongkyoung Chwa "Non linear Tracking Control of under actuate ship based on unified kinematic and dynamic model" , "Mediterranean Conference on control and automation", July-2007,Athina-Greece, IEEE 1-4244-1282x.

[6] Gokdeniz Neser,and Deniz Unsalan "Dynamics of ship and fenders during berthing in a time domain",Institute of Marine science and Technology ,Izmir ,Turkey , Ocean Engineering 33-(2006) 1919-1934.

[7] S.Sutulo, L.Moreira ,and C.Guedes "Mathematical models for ship path prediction in maneuvering simulation systems", Unit of Marine Technology and Engineering, University of Lisbon, Lisbon, Portugal, Ocean Engineering 29-(2002)1-19.

[8] Loh Sheau Ping "Hydrodynamic Coefficients of Maneuvering For Small Vessels", Faculty of Mechanical Engineering, University of Teknologi ,Malaysia-May(2004)

[9] A.Witkowska, M. Tomera, and R. Smierzchalski "Backstepping Approach to Ship Course Control”., Gdynia Maritime University ,Gdynia ,Poland., International Journal Appl. Math. Comput. Sci.,2007, Vol.17, No.1,73-85.

[10] Yuanhui Wang, Xiaocheng Shi, and Xinqian Bian "Modeling of Large Ship Motion and Design of Intelligent Rudder Control Mechanism” „Harbin Engineering University (HEU) "International Conference on Automation and Logistics" August 17-22, 2007 , Jinan , China, IEEE 1-4244-1531-4.

[11] Ming-Chung Fang , Jhih-Hang Luo "The Nonlinear hydrodynamic model for simulating a ship steering in waves with autopilot system", Taiwan, ROC ,Ocean Engineering 32(2005) 1486-1502.

[12] Yasuo Yoshimura "MMG mathematical model for maneuvering the ship motion" Workshop on Mathematical Models for Operations involving Ship-Ship interaction August 2005,Tokyo,Japan.

[13] Y. Yaving.,Zilman ,and Miloh "Ship Maneuverability in Finite Depth Water in the Vicinity of an Obstacle :A Stochastic Control Approach", Department of Electrical and Electronics, University of Pretoria, South Africa ,March (1994)-0898-1221(95)00158-1

[14] The Specialist Committee on Esso Osaka "Final Report and Recommendations", " the 23rd ITTC International Towing Tank Conference” Feb. 2002 Taejon, Korea 